\title{
Effects of 72 Hours Sleep Deprivation on Liver Circadian Clock Gene Expression and Oxidative Stress in Rats
}

\author{
Tang $\mathrm{Li}^{1}{ }^{*}$, Ruina Cao${ }^{1}$, Rui Xia ${ }^{*}$, Zhongyuan Xia ${ }^{2}$ \\ ${ }^{1}$ Department of Anesthesiology, The First Affiliated Hospital of Yangtze University, Jinzhou, China \\ ${ }^{2}$ Department of Anesthesiology, Renmin Hospital of Wuhan University, Wuhan, China \\ Email: *462244408@qq.com, ^879560350@qq.com
}

How to cite this paper: Li, T., Cao, R.N., Xia, R. and Xia, Z.Y. (2017) Effects of 72 Hours Sleep Deprivation on Liver Circadian Clock Gene Expression and Oxidative Stress in Rats. Yangtze Medicine, 1, 194-201.

https://doi.org/10.4236/ym.2017.14020

Received: September 27, 2017

Accepted: December 10, 2017

Published: December 13, 2017

Copyright $\odot 2017$ by authors and Scientific Research Publishing Inc. This work is licensed under the Creative Commons Attribution International License (CC BY 4.0).

http://creativecommons.org/licenses/by/4.0/

\begin{abstract}
Objective: To investigate the effects of 72 hours continuous sleep deprivation (SD) on circadian clock gene expression and oxidative stress in the rat liver. Methods: Twenty healthy male Sprague-Dawley rats were divided into 2 groups ( $\mathrm{n}=10$ each) using a random number table: normal control group (group C), sleep deprivation group (group SD). Group SD was treated with a modified multiple platform water environment method. After 72 hours sleep deprived, the levels of AST (Aspartate transaminase ) and ALT (Alanine aminotransferase) in serum were determined. The contents of malondialdehyde (MDA), the activities of superoxide dismutase (SOD) and glutathione peroxidase (GSH-Px) in the liver tissue of the rats were examined in both two groups. The expression levels of CLOCK, BMAL1 and CRY1 protein in liver tissue were examined by Western blotting. Results: Compared with group C, the content of MDA, and the levels of AST and ALT in serum were significantly increased $(\mathrm{P}<0.05)$; SOD activity was significantly decreased $(\mathrm{P}<$ 0.05); GSH-Px activity was significantly decreased $(\mathrm{P}<0.01)$; and the expression of CLOCK, BMAL1 and CRY1 protein was downregulated in group SD. Conclusion: 72 hours continuous sleep deprivation can downregulate the expression of circadian clock gene and promote oxidative stress in rats.
\end{abstract}

\section{Keywords}

Sleep Deprivation, Liver, Circadian Clock Genes, Oxidative Stress

\section{Introduction}

With the development of modern society and the industry competition, lack of sleep, insomnia and other sleep disorders are increasingly becoming a major 
public health problem. Sleep disorders not only increase the incidence of accidents in work, but also cause great harm to human health. Sleep deprivation affects many physiological functions, including organ hormone secretion, cognitive ability, memory and immune function. A considerable body of clinical evidence also suggests that sleep deprivation has a close relationship with the expression of circadian clock genes and oxidative stress [1].

Circadian rhythm is an important physiological process to maintain the homeostasis of organism. The circadian rhythm consists of a set of clock genes, such as CLOCK, BMAL, PER and CRY, which is regulated by the transcription-translational feedback loop composing of the biological clock gene and their encode protein. The CLOCK/BMAL complex activates the expression of PER and CRY. The activated PER and CRY in turn inhibit the activity of the CLOCK/ BMAL complex. In addition to this, recent biochemical and physiological studies have confirmed that sleep deprivation as a strong stressor for the body, increase the level of oxidative stress of the brain, resulting in cognitive dysfunction and memory loss [2].

Over the past decades, a mounting body of evidence has accumulated that sleep deprivation is associated with the development of many diseases (including cancer, neurological diseases and cardiovascular disease), but little is known regarding whether oxidative stress and circadian oscillations are important consequences of sleep deprivation in digestive system, especially in liver. As we all know, the liver is the main energy metabolism organ, and the liver can maintain energy homeostasis by regulating the expression of circadian clock gene to control the physiological activities [3]. Therefore, it is of great significance to explore the effects of sleep deprivation on liver injury. Here, in this study, we established a model of 72 hours sleep deprivation in rats to observe the effects of the long sleep deprivation on liver circadian clock gene expression and oxidative stress. Rats in group SD were sleep deprived for a 72-h period based on GU Ye, et al. [3].

\section{Materials and Methods}

\subsection{Materials}

SPF (specific pathogen free) grade male Sprague-Dawley rats were provided by Hubei Provincial Center for Disease Control and Prevention (Wuhan, China). MDA level, SOD and GSH-Px activities were measured by using commercial kits from Jiancheng Bio-engineering Institute (Nanjing, China). The RIPA buffer and bicinchoninic acid (BCA) assay kit were purchased from Beyotime Biotechnology (Shanghai, China.). The anti-CLOCK, anti-BMAL1, anti-CRY1 and anti- GAPDH antibody were purchased from Gene Tex (Texas, America).

\subsection{Animals and Sleep Deprivation}

All animal procedures were approved by the ethics committee of Wuhan University and were carried out in accordance with the NIH (National Institutes of 
Health) Guidelines for the Care and Use of Laboratory Animals. Twenty healthy male Sprague-Dawley rats, weighing $200-250 \mathrm{~g}$, aged $10-12$ weeks, were divided into 2 groups ( $\mathrm{n}=10$ each) using a random number table: normal control group (group C), sleep deprivation group (group SD). All rats were fed in a climate-controlled room $\left(22^{\circ} \mathrm{C}-24^{\circ} \mathrm{C}\right)$ on a $12 / 12$-h light-dark schedule (lights on at $7 \mathrm{AM}$ ) with a free access to food and water. All rats were allowed to acclimate for one week in the aquarium without sleep deprivation before starting the experiments.

Rats in group SD were sleep deprived for a 72 -h period using the columns-in-water model (modified multiple platform water environment method). Sleep consists of two main stages non-rapid eye movement (REM) and REM sleep. This method is reported to interfere with both non-REM and REM sleep, but mainly affect REM sleep. The cylindrical aquarium (diameter: $70 \mathrm{~cm}$, height: $40 \mathrm{~cm}$ ) contained 10 columns (platform diameter: $7 \mathrm{~cm}$, height: $8 \mathrm{~cm}$, with platform $1 \mathrm{~cm}$ above the water level), spaced $7 \mathrm{~cm}$ apart (edge to edge) [4]. The platforms arranged in two rows and rats could move from one platform to another. The water temperature maintained at $22^{\circ} \mathrm{C}-24^{\circ} \mathrm{C}$, normal $12 \mathrm{~h}$ light $/ 12$ $\mathrm{h}$ dark. Food and water were provided ad libitum, but they were woken up once entering into REM as a result of loss of muscle tone. The rats re-climbed the deprivation, and then falled into the water, that cycle repeated to sleep deprivation for 72 hs. Rats in group C were given to no treatment.

After 3 days, all rats were sacrificed, the liver tissues and body were weighed, and serum was collected for determination of liver function.

\subsection{Determined the Levels of AST and ALT in Serum}

Using the rate method to determine the levels of AST and ALT, the instrument was Modular P800 automatic biochemical analyzer (Roche), and its reagents, standards and quality control products were supported by Roche company too.

\subsection{Estimation of Liver Tissue Oxidative Stress}

All rats were sacrificed after three days of sleep deprivation. The liver samples were taken and washed with ice salt water. The liver tissue was weighed, $4^{\circ} \mathrm{C}$ homogenated, and then $3000 \mathrm{r} / \mathrm{min} 15$ min centrifuged. 10\% wet weight per volume liver tissue was prepared to determine the oxidative stress. The contents of MDA, the activities of SOD and GSH-Px in the supernatant were performed by assay kits according to the manufacturer's protocol.

\subsection{Western Blot Analysis}

The expression levels of CLOCK, BMAL1 and CRY1 were detected by Western blotting. The total protein was extracted by using RIPA lysis buffer (50 mM Tris, $\mathrm{pH} 7.4,150 \mathrm{mM} \mathrm{NaCl}, 1 \% \mathrm{NP}-40,0.5 \%$ sodium deoxycholate) and protease inhibitors were added immediately before use. The protein concentration of extracted samples was measured using the BCA protein assay kit. After adding 4 
times volume loading buffer, the simples were boiled for 10 minutes to denature. Sample protein containing $20 \mu \mathrm{g}$ total protein was subjected to SDS-PAGE using $12 \%$ resolving gel, followed by transfer to nitrocellulose membranes. After blocking with 5\% skimmed milk for 1 hour, the nitrocellulose was then incubated with anti-CLOCK, anti-BMAL1, anti-CRY1 and anti-GAPDH antibody (dilution 1:1000) overnight at $4{ }^{\circ} \mathrm{C}$, and then incubated with the secondary antibodies (dilution 1:5000). The bands were detected by the Odyssey image analyzer. The gray values of target proteins were used to reflect the level of expressions by software Image J.

\subsection{Statistical Analysis}

The data were reported as mean \pm standard deviation, analyzed using SPSS version 19.0 software, and compared by the unpaired Student $t$ test. Statistical significance was defined as $\mathrm{P}<0.05$.

\section{Results}

\subsection{The Effects of Sleep Deprivation on the Weights of Body and Liver Issue}

There was no significant difference between the groups before the experiment on the weights of body and liver $(\mathrm{P}>0.05)$. At the end of the experiment, the weights of body and liver of the rats in the sleep deprivation group were decreased than group $C$, and the differences were statistically significant $(P<0.05)$ (Table 1).

\subsection{The Effects of Sleep Deprivation on the Levels of AST and ALT in Serum}

At the end of the experiment, the levels of AST and ALT in serum of the rats in the sleep deprivation group were increased than group $\mathrm{C}$, and the differences were statistically significant $(\mathrm{P}<0.01)$ (Table 2$)$.

Table 1. Effects of sleep deprivation on the weights of body and liver issue $(\bar{x} \pm \mathrm{S}, \mathrm{n}=10)$.

\begin{tabular}{|c|c|c|c|c|}
\hline \multirow{2}{*}{ Group } & \multicolumn{2}{|c|}{ Body weight (g) } & \multicolumn{2}{|c|}{ Liver } \\
\hline & before & after & weight (g) & coefficient \\
\hline Group C & $242.7 \pm 13.2$ & $248.4 \pm 14.3$ & $10.01 \pm 2.02$ & $3.48 \pm 0.22$ \\
\hline Group SD & $238.9 \pm 11.5$ & $235.1 \pm 10.6^{*}$ & $8.43 \pm 1.76^{*}$ & $3.25 \pm 0.18$ \\
\hline
\end{tabular}

Compared with group $\mathrm{C},{ }^{\star} \mathrm{P}<0.05$.

Table 2. Effects of sleep deprivation on the levels of AST and ALT ( $\bar{x} \pm \mathrm{S}, \mathrm{n}=10)$.

\begin{tabular}{cccc}
\hline Group & AST $(\mathrm{U} / \mathrm{L})$ & ALT $(\mathrm{U} / \mathrm{L})$ & AST/ALT \\
\hline Group C & $166.4 \pm 38.3$ & $35.7 \pm 6.21$ & $4.50 \pm 1.13$ \\
Group SD & $244.2 \pm 70.1^{\star *}$ & $64.2 \pm 14.9^{* *}$ & $3.98 \pm 0.92$
\end{tabular}

Compared with group $\mathrm{C},{ }^{*} \mathrm{P}<0.01$. 


\subsection{The Effects of Sleep Deprivation on SOD, GSH-Px Enzyme Activity and MDA Level in Liver Tissue}

Compared with group $\mathrm{C}$, the content of MDA was significantly increased $(\mathrm{P}<$ $0.05)$, the activity of SOD was significantly decreased $(\mathrm{P}<0.05)$, the activity of GSH-Px was significantly decreased $(\mathrm{P}<0.01)$ in group SD (Figure 1$)$.

\subsection{The Effects of Sleep Deprivation on the Expression of CLOCK, BMAL1 and CRY1}

Compared with group C, the expression of CLOCK, BMAL1 and CRY1 protein were downregulated in group SD (Figure 2).

\section{Discussion}

Circadian rhythms refer to physiological processes that occur with a repeating period of approximately 24 hours, which are ubiquitously present in prokaryotes, fungi, algae, plants and mammals [5]. The clock gene in the body is critical for maintenance of homeostasis as well as adaptation to changing environmental conditions. Circadian rhythms evolve in almost all living liver organisms, which provide a relatively new perspective on hepatic function and metabolism, particularly with respect to sleep disorders and metabolic syndrome, finally leading to obesity, diabetes and dyslipidemia. Circadian regulation plays a large role in liver
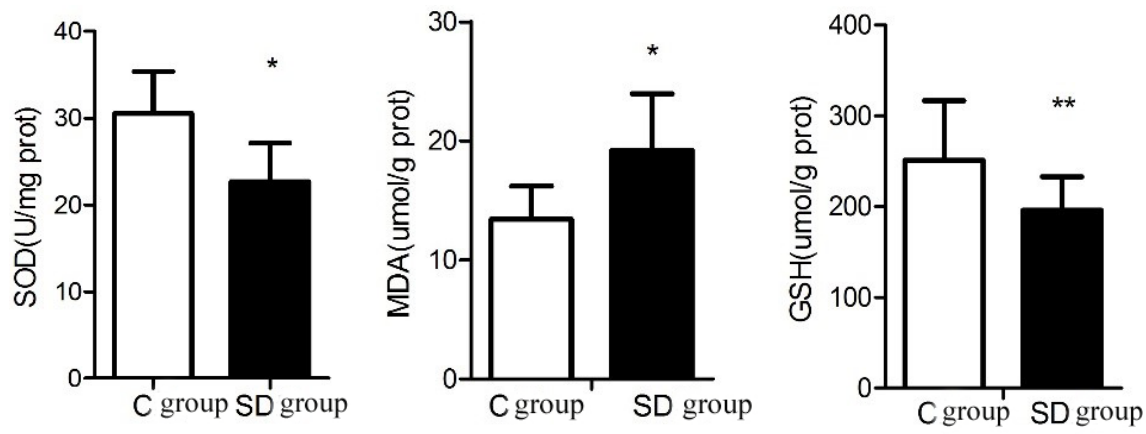

Figure 1. Effects of sleep deprivation on MDA, SOD and GSH levels in rat liver, Results are expressed as mean $\pm \mathrm{SD}$. Compared with group $\mathrm{C},{ }^{\star} \mathrm{P}<0.05,{ }^{* *} \mathrm{P}<0.01$.

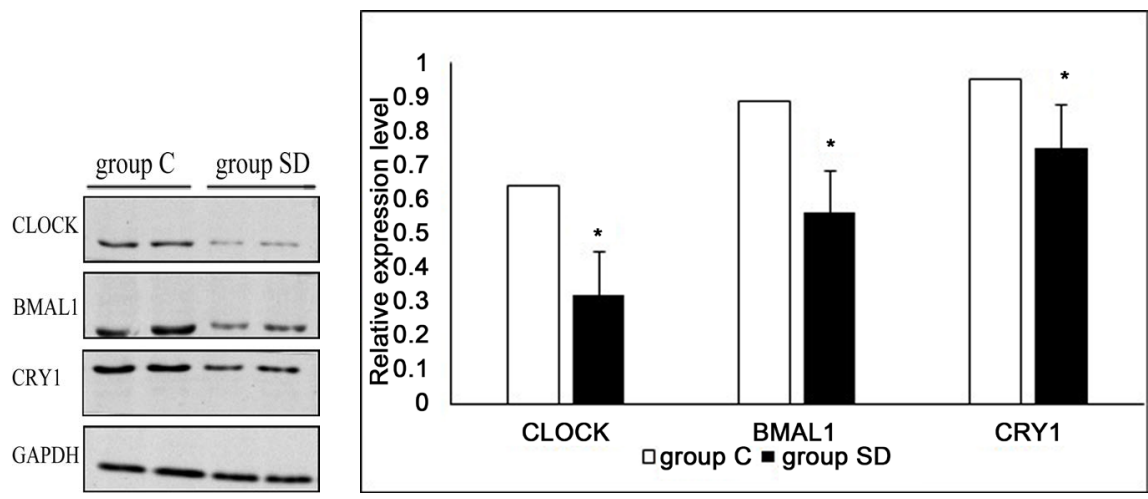

Figure 2. The expression levels of CLOCK, BMAL1 and CRY1 in the liver tissue of the two groups. Compared with group $\mathrm{C},{ }^{\star} \mathrm{P}<0.05$. 
metabolism, as glucose, bile acids, lipids, drug metabolism and enzyme expression and they are all subject to timed circadian control. Thus, liver local biological clock gene can maintain the liver homeostasis by regulating the absorption and metabolism of nutrients [6] [7].

Ferrell JM et al. found that even short-term destruction of the daily sleep pattern dramatically altered the balance of bile acids metabolism, and lipid homeostasis to contribute to dyslipidemia [8]. This was validated in our experiments. Remarkably, genetic or environmental disruption of the circadian clock can cause metabolic functions disorder such as food uptake, digestion, absorption, and detoxification [9]. Recent evidence indicates that the circadian clock can alter the levels of AST, ALT, and bile acids [10] [11]. This also was proved in our experiments.

Studies have shown that sleep deprivation is a strong oxidative stress response. Chang HM et al. found that sleep deprivation predisposed liver to oxidative stress and phospholipid damage [12]. Zhang YJ et al. found that three days after continuous sleep deprivation in rats, the contents of TSH, ACTH, CORT, were increased, and the longer times sleep deprived, the more contents increased [4]. When organism received stress stimulation, the hypothalamus-pituitary-adrenal cortex system activated to secrete catecholamines and glucocorticoid. The body produced a large number of reactive oxygen free radicals (ROS) in order to resist stress response, while the clearance ability was decrease. It has been suggested that accumulated ROS production and the consequent allostatic overload may lead to oxidative stress and subsequent lipid peroxidation, which may be responsible for the pathophysiological progressions of sleep deprivation-related liver damage. MDA as a cell wall peroxidation marker could be a good indicator for oxidative stress levels. Our experimental results showed that the content of MDA in liver tissue of sleep deprivation group was significantly higher than that of control group, which indicated that sleep deprivation could promote oxidative stress in rat liver. This was consistent with the results of Tang QJ et al. who detected serum MDA levels in 72 hours continuous sleep deprivation by a modified multiple platform techniques [13].

Besides producing increasingly, the scavenging ability reduction is another reason for ROS accumulation. SOD is among the most important members of the antioxidant defense system because of its special physiological activity. GSH-Px plays an important role in the antioxidant system because of its unique sulfhydryl group to accelerate the removal of free radicals in the body. In the liver tissue, GSH-Px also can accelerate the removal of free radicals to reduce liver injury. Everson et al. found that GSH-Px activity in the liver was significantly lower than that in the control group after 5 days continuous SD [14]. Our experimental results showed that the activity of SOD and GSH-Px in the liver of the rats in the group SD was significantly lower than that in the control group, indicating that sleep deprivation could reduce the antioxidant stress ability.

These changes were associated with widespread suppression of core clock 
gene expression and RNA rhythms of lipid metabolic, indicating the presence of sleep-clock-metabolism axis in liver, which mediated pathophysiology of sleep deprivation. We observed 72 hours continuous sleep deprivation in rats, and found that the expression levels of CLOCK, BMAL1 and CRY1 gene in liver tissue were significantly down-regulated by western blotting. It was confirmed that sleep deprivation could significantly affect the expression of biological clock gene in liver tissue, which resulted in the disorder of rhythmic biochemical reaction and the internal environment, leading liver injury.

Oxidative stress and biological clock genes are closely related. Recent studies have shown that several pathways for protecting cells from oxidative stress may be regulated by circadian rhythms, including genes involved in glutathione biosynthesis [15]. Krishnan et al. found that the circadian rhythm was critical to maintain a strong antioxidant defense in a study of the drosophila melanogaster [16]. Kondratova AA et al. found that clock-deficient mice could lead to oxidative damage and aging symptoms [17]. Hardeland $\mathrm{R}$ et al. suggested that the expressions of some antioxidant enzymes such as catalase, SOD and glutathione thiol transferase had circadian rhythm by microarray methods, and these rhythms could avoid excessive production of ROS, thus reducing biological macromolecular damage [18]. However, whether the molecular mechanism of the biological clock system disorder during sleep deprivation is associated with the signaling pathways of oxidative stress, does not know. Whether the production of ROS and the activity of the antioxidant enzymes are related with the sleepclock-metabolic axis are not yet known that need further study.

\section{References}

[1] Archer, S.N. and Oster, H. (2015) How Sleep and Wakefulness Influence Circadian Rhythmicity: Effects of Insufficient and Mistimed Sleep on the Animal and Human Transcriptome. Journal of Sleep Research, 24, 476. https://doi.org/10.1111/jsr.12307

[2] Ramanathan, L., Gulyani, S., Nienhuis, R., et al. (2002) Sleep Deprivation Decreases Superoxide Dismutase Activity in Rat Hippocampus and Brainstem. Neuroreport, 13, 1387-1390. https://doi.org/10.1097/00001756-200208070-00007

[3] Ye, G.U., Chen, X., Lun, S., et al. (2017) Effect of Continuous Sleep Deprivation on Liver Circadian Clock Gene Expression and Alteration of Liver Bio-Marker Levels in Rats. Military Medical Sciences, 41, 174-177.

[4] Zhang, Y.J. (2009) Effects of Sleep Deprivation on Rat Heart and Its Mechanism. Chinese Academy of Military Medical Sciences, 18, 224-226.

[5] Oster, H. (2015) CYP7A1: A Liver Circadian Clock Output Mediating the Metabolic Effects of Sleep Disruption. Cellular \& Molecular Gastroenterology \& Hepatology, 1, 574-575. https://doi.org/10.1016/j.jcmgh.2015.09.002

[6] Marcheva, B., Ramsey, K.M., Buhr, E.D., et al. (2010) Disruption of the Clock Components CLOCK and BMAL1 Leads to Hypoinsulinaemia and Diabetes. $\mathrm{Na}$ ture, 466, 627-631. https://doi.org/10.1038/nature09253

[7] Fujisawa, K., Takami, T., Kimoto, Y., et al. (2016) Circadian Variations in the Liver Metabolites of Medaka (Oryzias latipes). Scientific Reports, 6, Article Number 
20916. https://doi.org/10.1038/srep20916

[8] Ferrell, J.M. and Chiang, J.Y. (2015) Short-Term Circadian Disruption Impairs Bile Acid and Lipid Homeostasis in Mice. Cellular \& Molecular Gastroenterology \& Hepatology, 1, 664-677. https://doi.org/10.1016/j.jcmgh.2015.08.003

[9] Potter, G.D.M., Skene, D.J., Arendt, J., et al. (2016) Circadian Rhythm and Sleep Disruption: Causes, Metabolic Consequences, and Countermeasures. Endocrine Reviews, 37, 584-608. https://doi.org/10.1210/er.2016-1083

[10] Zhou, D., Wang, Y., Chen, L., et al. (2016) Evolving Roles of Circadian Rhythms in Liver Homeostasis and Pathology. Oncotarget, 7, 8625. https://doi.org/10.18632/oncotarget.7065

[11] Reinke, H. and Asher, G. (2016) Circadian Clock Control of Liver Metabolic Functions. Gastroenterology, 150, 574-580. https://doi.org/10.1053/j.gastro.2015.11.043

[12] Chang, H.M., Mai, F.D., Chen, B.J., et al. (2008) Sleep Deprivation Predisposes Liver to Oxidative Stress and Phospholipid Damage: A Quantitative Molecular Imaging Study. Journal of Anatomy, 212, 295. https://doi.org/10.1111/j.1469-7580.2008.00860.x

[13] Tang, Q.J., Tao, K.Z. and Miao-Miao, H.U. (2003) Oxidative Stress in 72 hs Sleep-Deprived Rat. Chinese Journal of Behavioral Medical Science, 12, 500.

[14] Everson, C.A., Laatsch, C.D. and Hogg, N. (2005) Antioxidant Defense Responses to Sleep Loss and Sleep Recovery. AJP Regulatory Integrative \& Comparative Physiology, 288, R374-R383. https://doi.org/10.1152/ajpregu.00565.2004

[15] Chow, E.S., Long, D.M. and Giebultowicz, J.M. (2016) Circadian Rhythm in mRNA Expression of the Glutathione Synthesis Gene Gclc Is Controlled by Peripheral Glial Clocks in Drosophila Melanogaster. Physiological Entomology, 41, 369. https://doi.org/10.1111/phen.12164

[16] Krishnan, N., Davis, A.J. and Giebultowicz, J.M. (2008) Circadian Regulation of Response to Oxidative Stress in Drosophila Melanogaster. Biochemical \& Biophysical Research Communications, 374, 299. https://doi.org/10.1016/j.bbrc.2008.07.011

[17] Kondratova, A.A. and Kondratov, R.V. (2012) The Circadian Clock and Pathology of the Ageing Brain. Nature Reviews Neuroscience, 13, 325. https://doi.org/10.1038/nrn3208

[18] Hardeland, R., Coto-Montes, A. and Poeggeler, B. (2003) Circadian Rhythms, Oxidative Stress, and Antioxidative Defense Mechanisms. Chronobiology International, 20, 921-962. https://doi.org/10.1081/CBI-120025245 